\title{
The effect of bone marrow mesenchymal stem cell transplantation on hypoxic pulmonary hypertension in rats
}

\author{
Hongjun Tian, Jingping Yang*, Xiuxiang Wang \\ The Third Affiliated Hospital of Inner Mongolia Medical University, Baogang Hospital, Baotou, Inner Mongolia, China
}

Received: September 11, 2018

DOI: $10.14725 /$ dcc.v5n4p17

\author{
Accepted: October 26, 2018 \\ Online Published: December 10, 2018 \\ URL: http://dx.doi.org/10.14725/dcc.v5n4p17
}

\begin{abstract}
Objective: To study the influence of bone marrow mesenchymal stem cells (MSCs) transplantation on hypoxic pulmonary hypertension (HPH) in rats.

Methods: MSCs in SD rats were separated, cultivated, identified in vitro, and labeled by the green fluorescence protein (GFP) adenovirus. Healthy male SD rats were randomly divided into four groups: normal control group (NC group) and HPH group, with eight rats in each group respectively; HPH + mesenchymal stem cell transplantation group (MSCs group) and HPH + vascular endothelial growth factor + mesenchymal stem cell transplantation group (VEGF + MSCs group), with twenty-four rats in each group respectively. In this experiment, intermittent normobaric hypoxia was employed to establish the pulmonary hypertension rat models, with stem cells transfected and transplanted. The mean pulmonary artery pressure (mPAP) was observed in rats to calculate the right ventricular hypertrophy index (RVHI); the morphological changes of pulmonary arterioles in each group of rats were observed under the microscope; the distribution and manifestation of MSCs fluorescently labeled by adenovirus transfection were observed in pulmonary arterioles under the fluorescence microscope at the set time points of $7 \mathrm{~d}$, $14 \mathrm{~d}$ and $28 \mathrm{~d}$ after the transplantation of stem cells.

Results: For NC group, the mPAP (mmHg) was $15.5 \pm 1.5$ at $28 \mathrm{~d}$, while the mPAP in HPH, MSCs and VEGF + MSCs groups were $26.1 \pm 1.9,21.6 \pm 2.7$ and $20.1 \pm 2.9$ respectively which were apparently higher than that in NC group $(p<.01)$. Compared with HPH group $(p<.01)$, the mPAP was obviously decreased in MSCs and VEGF + MSCs groups. There was no significant difference between MSCs and VEGF + MSCs groups. At $28 \mathrm{~d}$, RVHI for NC group was $0.28 \pm 0.02$, while the RVHI in HPH, MSCs and VEGF + MSCs groups were $0.43 \pm 0.07,0.34 \pm 0.03$ and $0.35 \pm 0.01$ respectively which were apparently higher than that in NC group $(p<.01)$. In comparison with HPH group, RVHI was significantly decreased in MSCs and VEGF + MSCs groups $(p<.05)$. There was no significant difference between MSCs and VEGF + MSCs groups. For HPH group, at $28 \mathrm{~d}$, pulmonary arterioles were apparently thickened, with luminal stenosis \& obliteration and incomplete endothelial cells. Compared with HPH group, pulmonary arterioles in MSCs group became thinning, with the lumen unobstructed and the integrity of endothelial cells improved. The changes in the manifestation of MSCs and VEGF + MSCs groups were not significant.

Conclusions: The transplantation of MSCs can improve the remodeling of pulmonary arterioles to partially reverse the progress of HPH; the combined transplantation of VEGF and MSCs doesn't improve the effect of MSC transplantation.
\end{abstract}

Key Words: Hypoxia pulmonary hypertension, Bone marrow mesenchymal stem cells, Stem cell transplantation, Vascular endothelial growth factor, Rats

\footnotetext{
${ }^{*}$ Correspondence: Jingping Yang; E-mail: yangron@ sina.com.cn; Address: The Third Affiliated Hospital of Inner Mongolia Medical University, Baogang Hospital, Baotou, Inner Mongolia, China.
} 
Hypoxic pulmonary hypertension (HPH), a common disease that seriously threatens human health, usually results from the hypoxic condition caused by chronic obstructive pulmonary disease (COPD). Hypoxia can disorder the endothelial function of pulmonary blood vessels, with the reactive constriction of pulmonary blood vessels enhanced, so that pulmonary blood vessels are remodeled to cause pulmonary hypertension. ${ }^{[1]}$

Mesenchymal stem cells (MSCs) were used as seed cells for tissue engineering, and vascular endothelial growth factor (VEGF) is a relatively strong, endothelial cell specific, positively regulatory factor that stimulates the formation of blood vessels, namely, it can promote the de novo formation of blood vessels. ${ }^{[2,3]}$ There are some different reports on the fact that the combined transplantation of VEGF and MSCs can enhance their effect on the remodeling and repair of pulmonary blood vessels. This study was designed to not only establish the HPH rat models, but also cultivate, separate, purify and identify bone MSCs, in addition, label MSCs with GFP adenovirus. ${ }^{[4-7]}$ In this experiment, labeled MSCs and VEGF + MSCs were transplanted by caudal vein in rats, in order to explore their effect on mean pulmonary arterial pressure (mPAP), right ventricular hypertrophy index (RVHI) and pathological changes of pulmonary arterioles in rats with $\mathrm{HPH}$.

\section{Materials and methods}

\subsection{Reagents and instruments}

GFP adenovirus and VEGF adenovirus were purchased from Shanghai Hanbio Biotechnology Co., Ltd., a self-made normobaric hypoxia cabin (equipped with an oxygen meter, an electromagnetic valve, a reduction valve, an electric fan) was also included. The measurement of pulmonary arterial pressure in rats was made by connecting YP100 type pressure transducer (Beijing Xinhangxingye Technology Trade Co., Ltd.) to four-channel physiological recorder (Chengdu Instrument Factory, China). The fluorescent inverted microscope (Leica, Germany) was used to observe the distribution of MSCs fluorescently labeled by adenovirus transfection in pulmonary arterioles. The flow cytometer (Becton, Dickinson and Company, USA) was applied to the identification of bone marrow MSCs in SD rats. CD44H PE, CD45 APC and CD90 FITC antibodies in rats were purchased from Nanjing Bioedify Biotechnology Co., Ltd.

\subsection{Experimental animals and grouping}

The experimental animals were provided by Experimental Center of Inner Mongolia University (Certification No.: Jingdongzi 8806R011), and they were 64 clean and health male SD rats, 6 weeks old, weighed 178-249 g. SD rats were randomly divided into four groups: normal control group
(NC group) and HPH group, with eight rats in each group respectively; MSCs group and VEGF + MSCs group, with twenty-four rats in each group respectively. Each group of rats had free access to food and water. There was no significant difference in body weight among groups $(p>.05)$.

\subsection{Establishment of the HPH rat models}

Intermittent normobaric hypoxia was applied to this experiment. ${ }^{[4]}$ In addition to NC group, the rats were placed in the self-made normobaric hypoxia cabin, in which the nitrogen was inlet. The oxygen concentration in the cabin was measured with an oxygen meter and reduced to $(10 \pm 1) \%$ within $60 \mathrm{~min}$. The anoxic condition was required to be maintained for $8 \mathrm{~h}$ every day, 6 days in a week, for 21 consecutive days.

\subsection{Primary culture, separation, purification, iden- tification and cell labeling of MSCs}

Bilateral femora were taken from 6-week old SD rats under the aseptic condition. MSCs in rat bone marrow were separated by use of density gradient centrifugation and adherence method, and then cultivated. 3 samples were randomly chosen from the cells in the second or third passage, and surface antigens of MSCs were measured with the flow cytometer, which were consistent with the positive expressions of CD44 and CD90. The negative expression of CD45 identified those cells as MSCs. After identification, MSCs were fluorescently labeled with GFP-adenovirus and GFPVEGF-adenovirus. Multiplicity of infection (MOI) ranged from 20 to 50. After $24 \mathrm{~h}$, the condition of cell transfection was observed under the fluorescent inverted microscope. When the transfection results met the requirements, these cells were made into GFP-labeled MSC suspension ( $24 \mathrm{ml})$ and GFP-VEGF-labeled MSC suspension (24 ml) for transplantation use..$^{[5,6]}$

\subsection{The transplantation of MSCs}

After the HPH rat models were established successfully, the rats in MSCs and VEGF + MSCs groups were slowly injected by caudal vein with $1 \mathrm{ml}$ of GFP-labeled MSC suspension and $1 \mathrm{ml}$ of GFP-VEGF-labeled MSC suspension (including $3 \times 10^{6}$ cells $/ \mathrm{ml}$ ) respectively. All the rats were fed in the same way as before. 8 rats were randomly chosen from MSCs and VEGF + MSCs groups at $7 \mathrm{~d}, 14 \mathrm{~d}$ and $28 \mathrm{~d}$, with all relevant indicators measured; for $\mathrm{NC}$ and $\mathrm{HPH}$ group, all indicators were measured at $28 \mathrm{~d}$. 


\subsection{The measurement of each indicator after the transplantation of MSCs}

\subsubsection{General observation}

Before and after the establishment of SD rat hypoxic models and the transplantation, it was required to observe the changes in general mental status, daily activity, food intake, the severity of hair loss and body weight of SD rats.

\subsubsection{The measurement of MPAP and RVHI}

After the rats were weighed at $28 \mathrm{~d}$, they were injected with $10 \%$ chloral hydrate $(0.6 \mathrm{ml} / \mathrm{kg})$ by abdominal cavity for anesthesia, with tracheal intubation performed. Besides, they were given intramuscular injection of succinylcholine chloride $(1.5 \mathrm{mg} / \mathrm{kg})$ to stop spontaneous breathing and given ventilation by HX-200 animal ventilator to adjust respiratory frequency to about 70 times per minute, with tidal volume of 6-8 $\mathrm{ml}$ and $\mathrm{I} / \mathrm{E}$ of 1:2. The right heart catheter was made of polyethylene capillary and inserted into the pulmonary artery through the jugular vein. Fourchannel physiological recorder was used to record mPAP. After SD rats were sacrificed, right ventricle (RV) and left ventricle plus septum $(\mathrm{LV}+\mathrm{S})$ were isolated and weighed to calculate RVHI.

\subsubsection{Pathological observation}

Pulmonary tissues were taken from the sagittal plane of lung hilus, dehydrated conventionally, embedded in paraffin, resected and stained with hematoxylin-eosin (HE). Under the light microscope, 3 fields of view were randomly chosen to observe the changes in the structure of pulmonary artery. The distribution of GFP-labeled MSCs and the thickness of the vascular wall of pulmonary arterioles were observed under the fluorescence microscope.

\subsection{Statistical treatment}

The experimental data were represented by mean \pm standard deviation $(\bar{x} \pm \mathrm{s})$, and SPSS 13.0 Statistical Software Package was applied to statistical analysis. Variance analysis was first performed for multiple groups of data. When the variance was homogeneous, one way ANOVA was applied to the comparison and $L S D$ was used in the test; when the variance was not homogeneous, Dunnett'T3 was used in the test.

\section{Results}

\subsection{Separation, cultivation and identification of MSCs in rats}

MSCs in rats were primary cultured for $24 \mathrm{~h}$ and most of the cells proliferated adherently. After 3 days, the cell colonies increased rapidly, expanded constantly and merged with each other. After 1 week, the bottom of the culture bottle was covered with cells, which were to be subcultured. In the third passage, most of the cells appeared to be fusiform, triangle, polygon and spindle, were uniformly and orderly distributed in fibroblastic style, and arranged in swirl (see Figure 1). After the transfection of cells with adenovirus, GFP-labeled MSCs in fusiform, triangle, polygon and spindle shape were observed under the fluorescent inverted microscope (see Figure 2). The results of the flow cytometer were: CD90 and CD44 were positive (the positive rates were $69.8 \%$ and $74.58 \%$ respectively), CD45 was negative (the positive rate was only $1.11 \%$ ). It was confirmed that the cultured and transfected cells were MSCs.

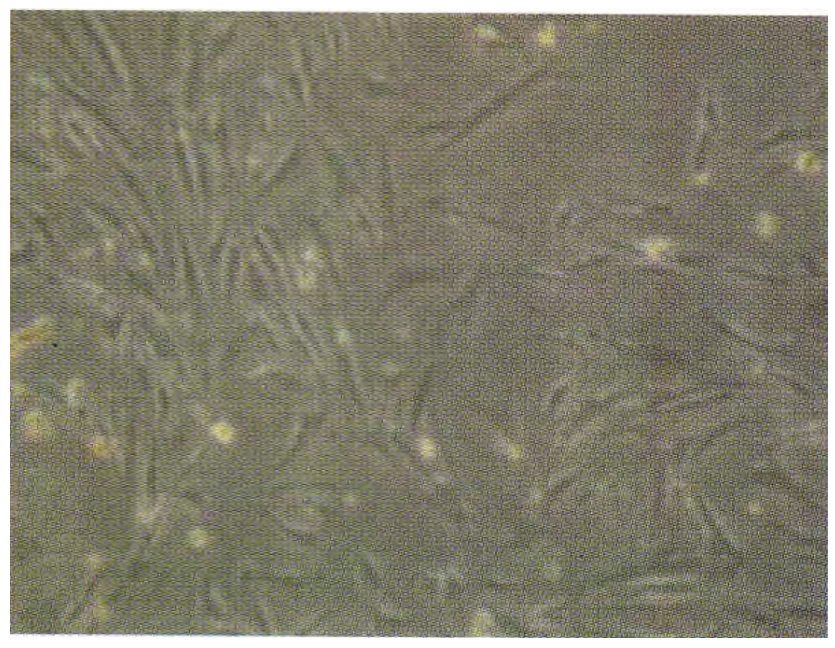

Figure 1: MSCs in the $3^{\text {rd }}$ generation of 3 days $(\times 100)$

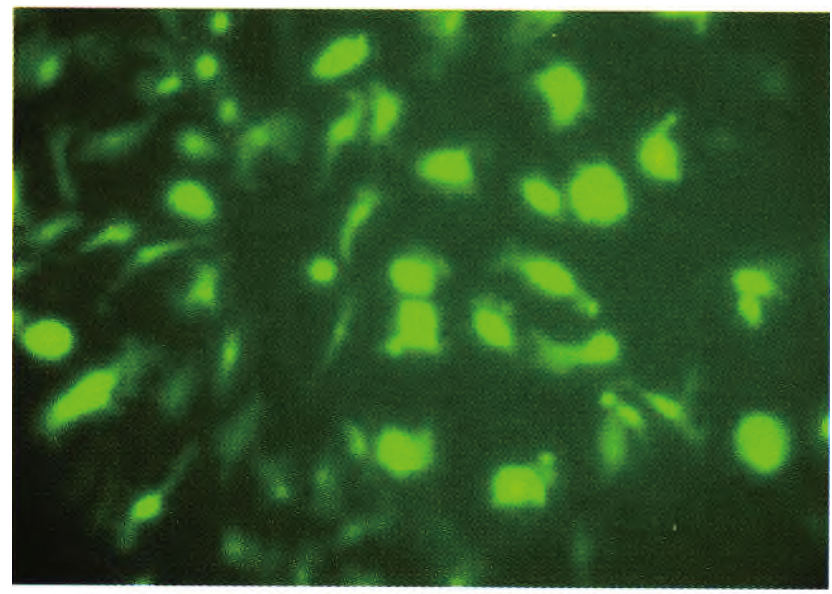

Figure 2: MSCs in the $3^{\text {rd }}$ generation, transfection images after 24 hours $(\times 400)$ 


\subsection{General condition of rats}

At $28 \mathrm{~d}$, the rats in NC group had regular hair color, no hair loss, flexible response, good food intake and weight gain. In HPH group, rats had severe hair loss, slow response, less food intake and reduced weight, which was apparently lower than that in $\mathrm{NC}$ group $(p<.01)$. After transplantation, hair loss was not obvious in rats of MSCs group, and the body weight was apparently increased than that in HPH group $(p<.01)$ and decreased than that in $\mathrm{NC}$ group $(p<.05$, see Table 1). After transplantation, hair loss was not obvious in rats of VEGF + MSCs group, and the body weight was apparently increased than that in HPH group $(p<.01)$ and decreased than that in NC group $(p<.05)$. However, there was no significant difference in body weight between MSCs group and VEGF + MSCs group $(p>.05$, see Table 1).

\section{3 mPAP and RVHI}

In NC group, mPAP and RVHI were respectively $(15.5 \pm$ 1.5) $\mathrm{mmHg}$ and $0.28 \pm 0.02$. In HPH group, mPAP and RVHI were $(26.1 \pm 1.9) \mathrm{mmHg}$ and $0.43 \pm 0.07$ respectively, which were significantly higher than those in NC group $(p<.01)$, indicating the successful establishment of HPH models. In MSCs group, mPAP and RVHl were respectively $(21.6 \pm 2.7) \mathrm{mmHg}$ and $0.34 \pm 0.03$, which were obviously higher than those in NC group $(p<.01)$, but significantly lower than those in HPH group $(p<.01, p<.05)$, suggesting that the transplantation of stem cells can partially improve pulmonary hypertension and RVHI. In VEGF + MSCs group, mPAP and RVHl were respectively (20.1 \pm
2.9) $\mathrm{mmHg}$ and $0.35 \pm 0.01$, which were obviously higher than those in NC group $(p<.01)$, but significantly lower than those in HPH group $(p<.01, p<.05)$. However, there was no significant difference in mPAP and RVHI between MSCs group and VEGF + MSCs group $(p>.05$, see Table 2).

Table 1: Comparison in body weight among four groups of rats before hypoxia and at $28 \mathrm{~d}$ after hypoxia stem cell transplantation $(\mathrm{g}, \bar{x} \pm \mathrm{s}, \mathrm{n}=8)$

\begin{tabular}{lll}
\hline Group & Before hypoxia & $\mathbf{2 8 ~ d}$ \\
\hline NC & $216.0 \pm 22.9$ & $318.8 \pm 24.2^{\# \#}$ \\
HPH & $218.9 \pm 24.7$ & $261.0 \pm 19.7^{* *}$ \\
MSCs & $208.9 \pm 24.8$ & $296.1 \pm 18.5^{* \#}$ \\
VEGF + MSCs & $216.3 \pm 19.9$ & $295.8 \pm 18.8^{* \#}$ \\
\hline
\end{tabular}

Note. NC: Normal control group; HPH: Hypoxia pulmonary hypertension; MSCs: Bone marrow mesenchymal stem cells; VEGF: Vascular endothelial growth factor; ${ }^{*} p<.05,{ }^{* *} p<.01$ vs. NC group; ${ }^{*} p<.05,{ }^{\#} p<.01$ vs. HPH group

Table 2: The changes in mPAP and RV/LV + $\mathrm{S}$ in four groups of rats with hypoxia and stem cell transplantation $(28 \mathrm{~d})(\bar{x} \pm \mathrm{s}, \mathrm{n}=8)$

\begin{tabular}{lll}
\hline Group & $\mathrm{mPAP}(\mathrm{mmHg})$ & $\mathrm{RV} / \mathrm{LV}+\mathrm{S}$ \\
\hline $\mathrm{NC}$ & $15.5 \pm 1.5^{\# \#}$ & $0.28 \pm 0.02^{\# \#}$ \\
HPH & $26.1 \pm 1.9^{* *}$ & $0.43 \pm 0.07^{* *}$ \\
MSCs & $21.6 \pm 2.7^{* * \#}$ & $0.34 \pm 0.03^{* * \#}$ \\
VEGF + MSCs & $20.1 \pm 2.9^{* * \#}$ & $0.35 \pm 0.01^{* * \#}$ \\
\hline
\end{tabular}

Note. NC: Normal control group; HPH: Hypoxia pulmonary hypertension; MSCs: Bone marrow mesenchymal stem cells; VEGF: Vascular endothelial growth factor; ${ }^{* *} p<.01$ vs. NC group; ${ }^{\#} p<.05,{ }^{\#} p<.01$ vs. HPH group
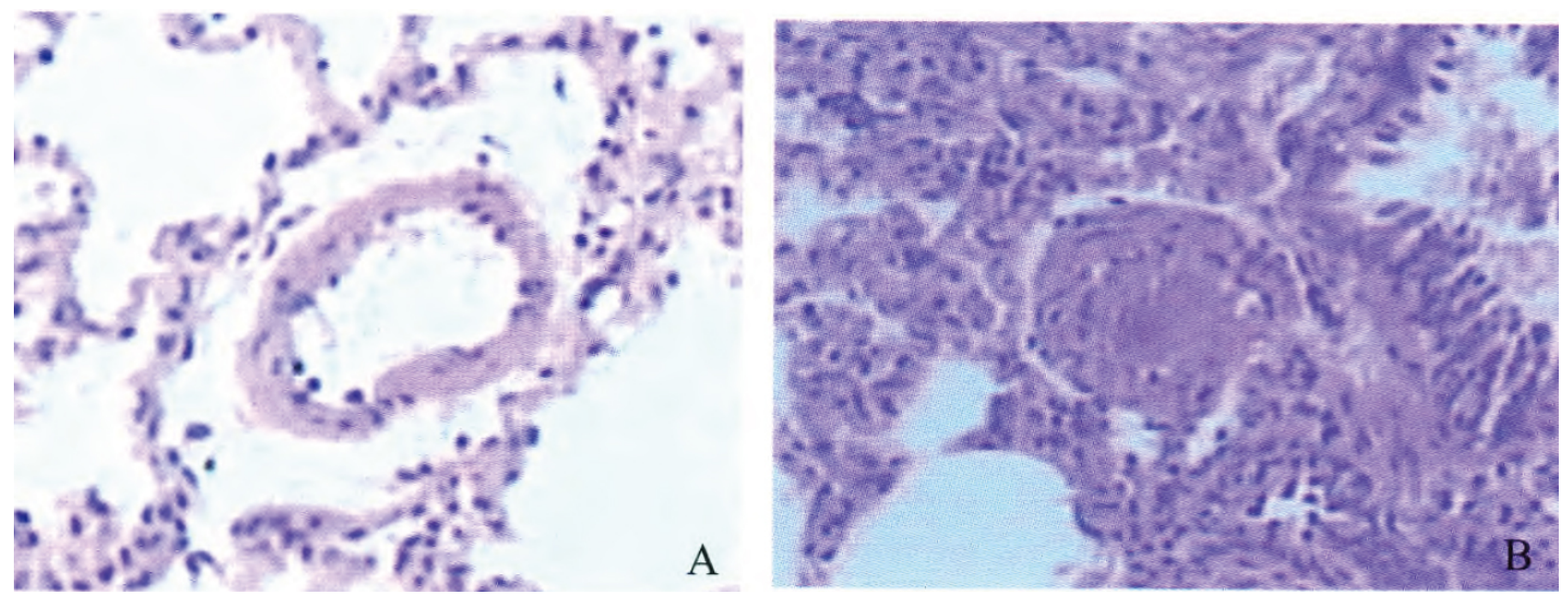

Figure 3: Morphology of pulmonary artery hypertension in each group of rats $(\mathrm{HE} \times 100)$

A: Normoxia group; B: Hypoxia group 


\subsection{The microscopic observation of pulmonary blood vessels in rats}

Under the microscope, the pulmonary arterioles in NC group became thinning, with the lumen unobstructed, and endothelial cells were complete and continuous (see Figure $3 \mathrm{~A})$. At $28 \mathrm{~d}$, in HPH group, it was visible that the pulmonary arterioles became thickened, with luminal stenosis $\&$ obliteration and incomplete endothelial cells (see Figure 3B). At $7 \mathrm{~d}$ after transplantation, in MSCs group, it was observed that the labeled MSCs migrated into the pulmonary arterioles under the fluorescence microscope. The vascular wall and the lumen were partially improved in comparison with HPH group (see Figure 4A), and further improved at $14 \mathrm{~d}$ (see Figure 4B). At $28 \mathrm{~d}$, the pulmonary arterioles became thinning, with the lumen unobstructed, which was further improved in comparison with $7 \mathrm{~d}$ and $14 \mathrm{~d}$ (see Figure 4C). There was no significant difference between VEGF + MSCs group and MSCs group. See Figure 5A, 5B and 5C for the manifestation of VEGF + MSCs group at $7 \mathrm{~d}, 14 \mathrm{~d}$ and $28 \mathrm{~d}$ under the fluorescence.
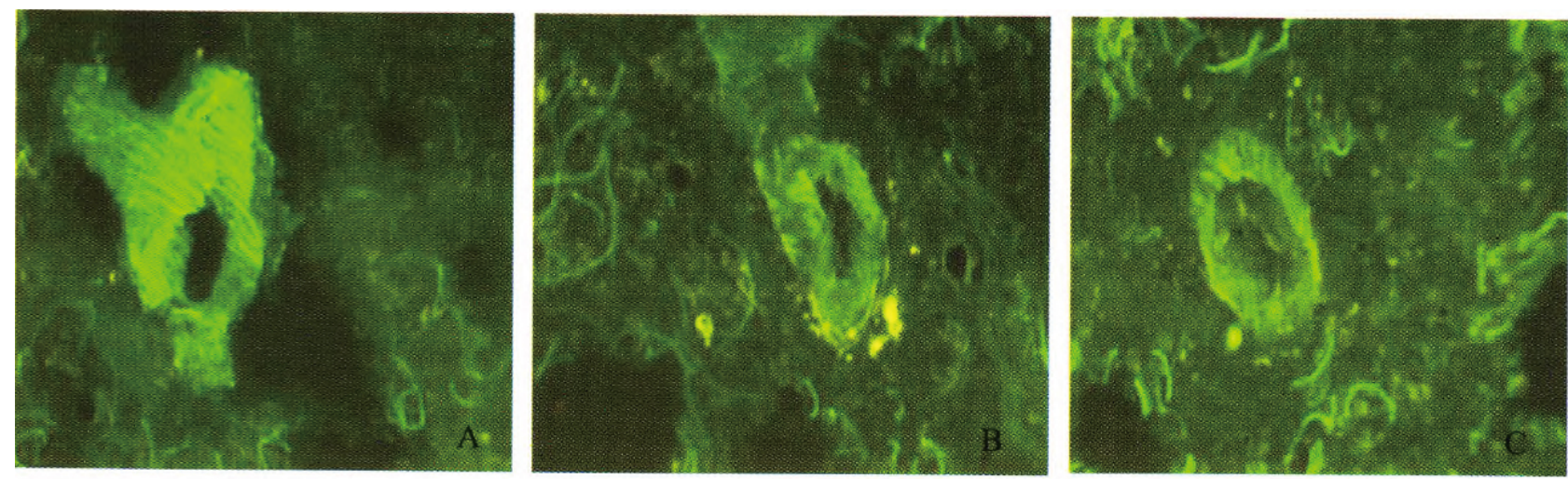

Figure 4: Morphology of pulmonary artery hypertension in each group of rats (adenovirus transfection fluorescently labeled $\times 400$ )

A: MSCs transplantation after 1 week; B: MSCs transplantation after 2 weeks; C: MSCs transplantation after 4 weeks
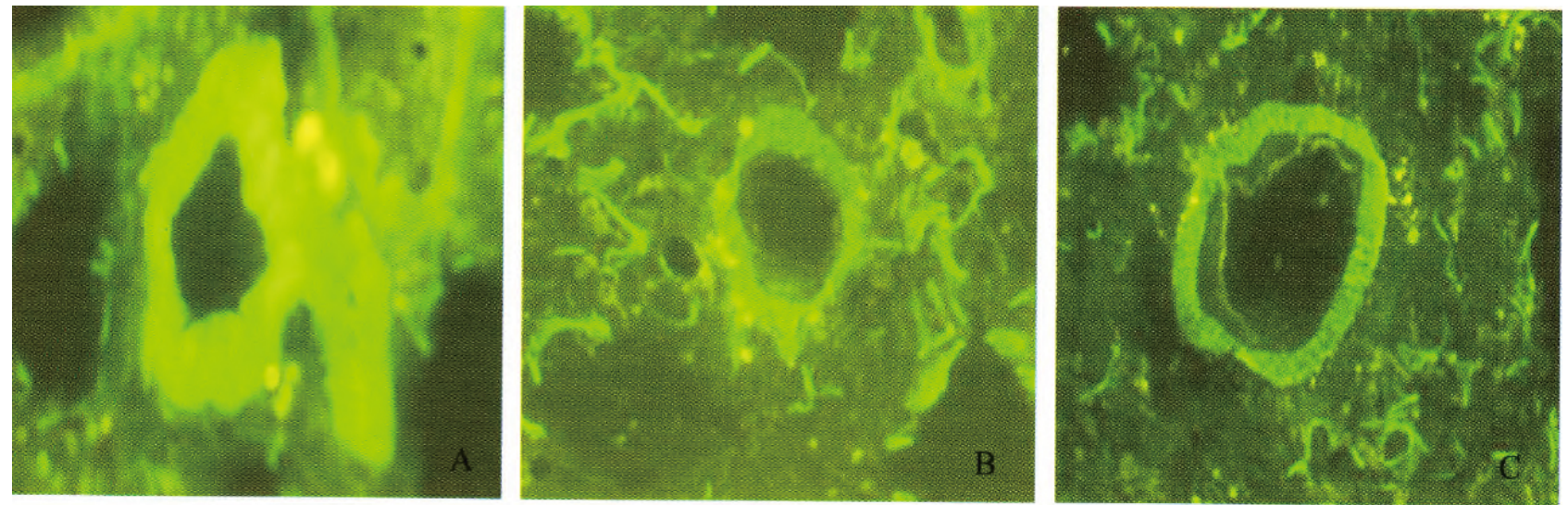

Figure 5: Morphology of pulmonary artery hypertension in each group of rats (adenovirus transfection fluorescently labeled $\times 400$ )

A: VEGF + MSCs transplantation after 1 week; B: VEGF + MSCs transplantation after 2 weeks; C: VEGF+ MSCs transplantation after 4 weeks

\section{Discussion}

HPH occurs in the process of pulmonary vasoconstriction and pulmonary remodeling caused by hypoxia due to altitude sickness and COPD, with a high incidence. It seriously affects patients' survival and life quality. Currently, there are no effective and safe drugs for pulmonary hypertension. Bone marrow MSCs transplantation is a new technology emerging in recent years. This type of cells, derived from mesoderm, are high potential seed cells which are of high proliferation, self-renewal and multi-directional differentiation. The reason why these cells are considered as seed 
cells is that they have a characteristic of multi-directional differentiation and in-vitro expression of various exogenous genes. ${ }^{[7]}$ Besides, MSCs may have an effect on repairing damaged pulmonary blood vessels and relieving pulmonary arterial pressure, which makes this technology become a method for the treatment of pulmonary hypertension. In this experiment, the transplantation of MSCs was applied to the treatment of HPH in rats. It was found that after transplantation, the general condition of the rats was better than that in HPH group, but mPAP and RVHI were obviously lower in MSCs group and VEGF + MSCs group than those in HPH group. In addition, there was no significant difference in MPAP and RVHI between MSCs group and VEGF + MSCs group. At $7 \mathrm{~d}$ after transplantation, in MSCs group, it was observed that the labeled MSCs migrated into the pulmonary arterioles under the fluorescence microscope or the optical microscope. The vascular wall and the lumen were partially improved in comparison with $\mathrm{HPH}$ group, and further improved at $14 \mathrm{~d}$. At 28 day, the pulmonary arterioles became thinning, with the lumen unobstructed, which was further improved in comparison with $7 \mathrm{~d}$ and $14 \mathrm{~d}$. There was no significant difference between VEGF + MSCs group and MSCs group, which was similar to the above study. It is indicated that the transplantation of MSCs can not only dilate, reverse and repair damaged pulmonary blood vessels, but also reduce the remodel-

\section{References}

[1] Cui H, Wei ZM, Fan L, et al. Clinical predictive value of hemoglobin level in mortality of hospitalized COPD patients. Chinese Journal of Applied Physiology. 2012; 28(5): 394-397.

[2] Chen S, Dai B, Zhu FM, et al. The effect of MSCs on differentiation of CB CD34+ cells into megakaryocytes. Chinese Journal of Applied Physiology. 2008; 24(1): 77-80.

[3] Zhang P, Dong L, Yan K, et al. CXCR4-mediated osteosarcoma growth and pulmonary metastasis is promoted by mesenchymal stem cells through VEGF. Oncol Rep. 2013; 30(4): 1753-1761. PMid: 23863999. https ://doi.org/10.3892/or. 2013.2619

[4] Yang JP, Li HZ, Qi HW. A Study of the effects of L-arginine in pathogenesis of hypoxic pulmonary hypertension. Chinese Journal of Applied Physiology. 2000; 16(4): 350-353. ing of pulmonary blood vessels in HPH rats and effectively treat $\mathrm{HPH}$ in rats, providing a new idea for the treatment of pulmonary hypertension. VEGF is a polypeptide growth factor that can synthesize and release nitric oxide ${ }^{[8]}$ to improve pulmonary artery pressure; promote the division of endothelial cells and the proliferation of angiogenic factors to improve pulmonary blood vessels; inhibit multiple factors that cause the apoptosis of endothelial cells; and increase the tolerance of endothelial cells to superoxide. However, this experiment shows that the effect of the combined tranplantation of VEGF and MSCs on HPH in rats is not obvious, and the specific mechanism needs to be further studied.

The transplantation of MSCs can reduce HPH and partially reverse the progress of $\mathrm{HPH}$, which can be used as a new measure to treat pulmonary hypertension. However, the following problems still remain to be solved: the survival time and differentiation ability in the host after transplantation, and the safety of transplantation need a further research for solution. The treatment of HPH by the transplantation of MSCs will be promising if the corresponding problems are solved.

\section{Conflicts of Interest Disclosure}

The authors have no conflicts of interest related to this article.

[5] de Mattos Carvalho A, Alves AL, Golim MA, et al. Isolation and immunophenotypic characterization of mesenchymal stem cells derived from equine species adipose tissue. Vet Immunol Immunpathol. 2009; 132(2-4): 303-306. PMid: 19647331. https : //doi.org/10.1016/j.vetimm.2009.06.014

[6] Morikawa S, Mabuchi Y, Kubota Y, et al. Prospective identification, isolation, and systemic transplantation of multipotent mesenchymal stem cells in murine bone marrow. J Exp Med. 2009; 206(11): 2483-2496. PMid: 19841085. https://doi.org/10.1084/jem. 20091046

[7] Haider M, CappeIlo J, Ghandehari H, et al. In vitro chondrogenesis of mesenchymal stem cells in recombinant silk-elastinlike hydrogels. Pharm Res. 2008; 25(3): 692-699. PMid: 17404809. https://doi.org/10.1007/s11095-007-9282-8

[8] Bates DO. Vascular endothelial growth factors and vascular permeability. Cardiovasc Res. 2010; 87(2): 262-271. PMid: 20400620. https://doi.org/10.1093/cvr/cvq105 